\title{
A Framework Used for Analysis of User Experience in Games
}

\author{
Vítor Carvalho [D [ University of Fortaleza | carva03@edu.unifor.br ] \\ Elizabeth Furtado (iD [ University of Fortaleza | elizabet@unifor.br ]
}

\begin{abstract}
The gaming industry has grown considerably in the last decades, designing experiences and interactive platforms through games, a popular culture entertainment medium. With the advancement of technologies and user experience design approaches, a challenge is faced for the constant improvement of gameplay aspects aiming to improve pleasurability and immersion perceived by its users. To face this challenge, we designed a conceptual framework named GLIMPSE (acronym built to represent its main elements: Goals, Learning, IMmersion, PreSEnce, Context) used for analysis of user experiences with games. The constructs were found within the literature. A questionnaire was developed to validate the framework's design through collecting user opinions, gathering 241 valid responses from gaming forums and community users. Further analysis shows the elements and sub-elements designed within GLIMPSE are found in many games and the experiences they exert. Another important result from analysis reveals significant statistical data highlighting gender-wise differences from participants' opinions.
\end{abstract}

Keywords: User experience, game design, games user research, conceptual framework, evaluation.

\section{Introduction}

Games predate from ages where humans have come together to socialize and articulate fun into their environments (Kumar \& Weger, 2013). Amongst talks and intellectual exchanges, rolling dice, or crafting pins to symbolize characters and carving boards on the ground would make for the first civilization games (Kumar \& Weger, 2013). Recognizing shapes, decision-making processes, and testing mental models have helped draw a learning curve from game newbies to masters (Desurvire \& Wiberg, 2010; Isbister, 2010; Hodent, 2017).

Games have been known to enhance motor and cognitive skills as players train and develop their abilities through trial and error (Desurvire, Caplan, \& Toth, 2004; Desurvire \& Wiberg, 2010; Hodent, 2017). It also evolved to allow much more control from players and to give back much more than just pure observational real-world inquiries (Desurvire \& Wiberg, 2010; Hodent, 2017; Kumar \& Herger, 2013). This entertainment medium is heavily sustained by businesses such as SONY, Microsoft, and Nintendo, having developed video gaming consoles in the previous decades with worldwide selling surpassing millions of units (Sirani, 2019), clarifying the ultimate impact these devices have in delivering gaming experiences.

Although games are built for entertainment, it does not always render pleasing situations. Trial and error allow for learning to be established. However, games may not be designed to sustain the process, failing its players when, for instance, it does not aid recovery from mistakes (Hodent, 2017), it does not follow the player's abilities when facing overwhelming experiences where the challenge is higher than what the player can endure (Takatalo, Häkkinen, \& Nyman, 2015), it fails to introduce players to the game's mechanics (Desurvire \& Wiberg, 2010). Faults like these can often frustrate players and drive them out of the designed experience, increasing the chances of potentially losing their interest.
Games can be designed with a wide variety of themes and objectives, to build experiences in fostering player interest and to reach a broad consumer audience. Aspects such as budget, development time, and team size can elevate a game to the triple A level, an informal terminology used within the games industry to designate projects with significant development and marketing budgets (Tamassia et al., 2016).

In addition, machines have been advancing faster than the human brain, making it a constant to be studied in the development of any artifact for human interaction, a science widely considered by the Human-Computer Interaction (HCI) field (Nacke, 2013, 2015). Other fields such as neuroscience, addressing psychological and cognitive aspects, and user research field, discussing the players' perceived gaming experiences (Nacke, 2013, 2015; Hodent, 2017), have fostered work to aid game design.

The mentioned fields present frameworks, game design principles, and game usability guidelines to help developers conceive and evaluate game designs for humans. They consider brain limitations (i.g.: attention, memory, focus) (Hodent, 2017), and propose guidelines for game design, such as establishing the learning process, allowing novice players to learn and master its mechanics (Desurvire \& Wiberg, 2010).

Considering that the technologies and design processes for creating games evolve quickly, bringing new application contexts and, consequently, new challenges, we created a framework to update the literature and to guide game developers in identifying the necessary constructs to the design experiences with games.

This paper's objectives are: i) to identify and categorize the User eXperience (UX) and HCI constructs through literature review; ii) to propose a framework; and finally: iii) to validate the framework.

The proposed framework, designed as a tool to assist game designers, consists of elements and sub-elements exploring UX constructs in game design. The GLIMPSE framework has the main elements: Presence and Interaction, Goals, Learning, Context, and Immersion. These elements, 
in turn, are made up of sub-elements, and so forth. For example, the Learning element consists of the influencing sub-elements, onboarding, and sense of owning, which are divided into more sub-elements.

To validate the framework's usefulness driven by 5 validation objectives were defined after its main elements. With main inspiration in post-test questionnaires commonly used in playtests, a questionnaire was designed. With 241 valid responses and further analysis, was conducted to extract relevant data between age and gender groups.

We then proceed to discuss each validation objective according to the results and analysis, having satisfied all 5 validation objectives. Our analysis points GLIMPSE elements are indeed found and important to be validated in game design, such as creating believable and relatable virtual entities portraying humanlike behavior, creating and sustaining learning experiences to introduce players to game mechanics and reward them for completing goals to enhance their experience. Our results and analysis also found gender disparities between certain framework elements, especially the presence and interaction element, which women have shown preference in playing alone instead of groups of players.

This article is structured in the following manner: Sections 2 presents the reviewed literature used as foundation to the work presented in section 3: the GLIMPSE framework. In section 4, we present the validation method. The statistical results gathered from the questionnaire are in section 5 , followed by further analysis in section 6 . We then proceed to discuss our findings in section 7 and conclude at section 8 .

\section{User Experience in Games}

This section presents the literature used as foundation to design our framework, firstly introducing the UX and Games User Research (GUR) fields to pave our understanding to the further subsection, presenting the reviewed literature for constructs considered for designing and evaluating gaming experiences.

\subsection{User Experience}

The UX concept has become prominent within the HCI field, accounting for experiences made during the interaction between the user and a product (Bernhaupt, 2010). Gaming experiences are exerted beyond simple tasks with long invested periods of time. As an interactive medium, it joins many design alternatives to convey immersive experiences specific to its niche, where players are at the center of game design.

When going through experiences, players are often motivated through personal, contextual, and social objectives (Isbister, 2010), while capturing the world's aspects through their perspective in the given moment. An experience is defined by considering cognitive abilities (Nacke, 2015; Hodent, 2017), the context, and the environment the user is (Isbister, 2010; Cohen \& Newton Fernando, 2009), how aesthetically pleasing the experience is perceived by the user (Calvillo-Gámez, Cairns, \& Cox,
2015) and social influence with relatives (Isbister, 2010; Oulasvirta, 2009; Cohen \& Newton Fernando, 2009). These elements guide developers to design pleasurable and enjoyable experiences for their customers.

Developers must delve themselves into the frustrations and necessities the customers experience to grasp what issues the players are facing, as thoroughly as possible. Games and interactive experiences are built upon the premise of delivering outstanding virtual worlds depicting the most varied genres and stories to the hands of the player while enabling the sense of being a protagonist (Desurvire \& Wiberg, 2010; Isbister, 2010; Hodent, 2017).

Being a cultural feature and popular media, games can be seen in many contexts and devices such as tabletop games with dice and pins, on consoles and personal computers, offering highly immersive gaming experiences, and smartphones, more portable and accessible (Isbister, 2010). The experience players have while playing games is often called gameplay.

Games can convey learning principles, social interactions (physically and virtually), goal elaboration, virtual ambiances, narratives, sounds effects, instant feedback, and embedded systems to immerse the user into the game (Desurvire \& Wiberg, 2010; Hodent, 2017; Champion, 2011). The immersion concept is often allied with the flow concept (Csikszentmihalyi, 1990), where the players find themselves in a flow state meaning their abilities match the faced in-game challenge, representing the optimal experience.

The GUR field characterizes game developers approaches for research, fieldwork, interviews, and data collection to support their design processes. Although a triple A game is designed with a large budget and a long development period, development studios have time and monetary constraints impacting user research processes adoption.

An example of GUR in action are playtests, test sessions generally aimed at assessing the game's ability to sustain pleasurable experiences, and if the game is meeting certain criteria. It can be approached through behavioral observation, questionnaires, interviews, focus groups, card sorting, among others (Nacke, 2013; Hodent, 2017). These methods measure psychological aspects of user interaction with games, can help to improve the User Interface (UI), fine-tune in-game interactions, improve visual or audible feedback, assess whether accessibility features are being effective, among others (Hodent, 2017), all before it is set to launch.

\subsection{Literature Review}

\subsubsection{Presence and Awareness in Virtual Environments}

A game also portrays a virtual environment that the player interacts with. It can be a vast and big forest with a character roaming through it, or it can just be a two-dimensional game. According to the literature, the concept of presence (Takatalo, Häkkinen, \& Nyman, 2015; Cohen \& Newton Fernando, 2009) employs "the sense of being there", even if said environment is entirely virtual. This connection is 
characterized by the player's sense of self-presence in the virtual environment. The sense of self-presence is reinforced when the player's wills and actions can be exerted within the game's domain of acceptance and are portrayed accordingly. Although presence and immersion seem to be connected, they do not mean the user is having an enjoyable experience (Calvillo-Gámez, Cairns, \& Cox, 2015).

Presence relates to awareness, as it stands for the player being conscious of their surroundings. It not only encompasses the ambiance the user is physically located, but it goes beyond it by also tackling a sense of identity, activities, and neighbors as well in the virtual ambiance. Notably, awareness can be addressed by three different types, as described by Cohen and Fernando (2009): presence, telepresence, and co-presence. As the name suggests, presence defines one's feeling of being part of the virtual environment. Telepresence defines the feeling of being in control of remotely located systems, in which the player is fully aware such a system is located elsewhere. Moreover, co-presence represents the shared sense of presence among a group of people and the current virtual environment this group is located in. Co-presence is mostly represented in triple A games as multiplayer activities where players connect through their devices to play together, either cooperatively or competitively.

Games are often backed up by Non-Player Characters (NPC), which are virtual entities controlled by the game, responding to commands prompted by real players, and controlled by Artificial Intelligence (AI). These entities are programmed to mimic human behavior, establishing social connections through "unconscious subliminal attributions due to human-like social cues, outward appearance, and corresponding behavior (even if the user is aware that the agent is not a real person), and is reinforced by the immersion into the game world and the related suspension of disbelief." (Emmerich et al, 2018), connecting to the concept of co-presence coined by Cohen and Fernando (2009). In Figure 1, a screenshot from the game Destiny 2, shows an NPC named Lord Shaxx is approachable to interact with the player. Lord Shaxx is designed to be the front-page for player-vs-player activities inside the Destiny 2 game, offering access to goals, namely quests and bounties, alongside rewards obtainable through in-game currency exchange. It visually resembles a human being and acts like one, portrayed with movements and voice.

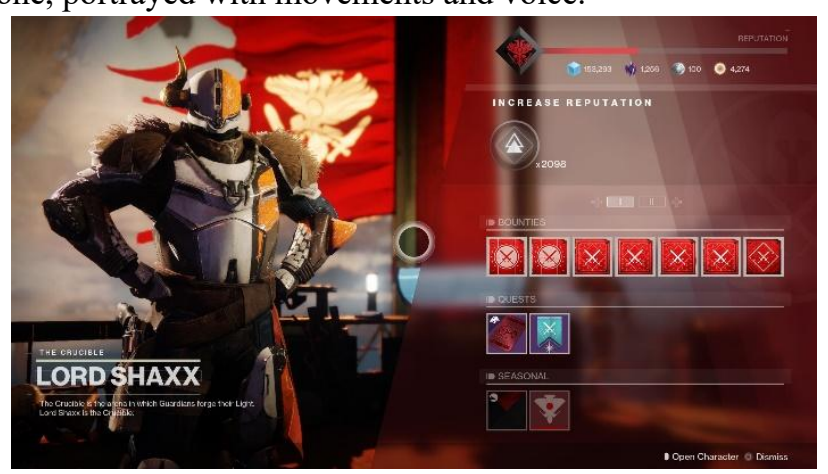

Figure 1. The NPC Lord Shaxx. Destiny 2 (Bungie).
"People and objects may have locations in and relationships to both physical space and one or more virtual spaces, and these different spaces together interact to constitute overall system behavior and user experience." (Cohen \& Newton Fernando, 2009). Both real and virtual environments can coexist and have their characteristics overlap in many situations.

\subsubsection{Player Empowerment}

A clear definition of the gaming experience stands in the one-to-one relationship established between the game and the player, portraying this and only this connection (CalvilloGámez, Cairns, \& Cox, 2015), leaving aside from any external factors to the average gaming scenario. CalvilloGámez e his colleagues define the concept of puppetry, a set of characteristics applied in games to evaluate the interaction players have over the game's virtual environment and how well the connection between the two is when the game creates an experience that resembles the outcomes of players' actions.

Puppetry subdivides into three subsets, namely control, ownership, and facilitators. Control applies to the activities and actions the game has in store for the players, letting them manipulate available resources while responding to their desired actions, enabling the players to own the game by influencing outcomes. Small actions represent actions players perform such when pressing buttons while understanding how to control the game through its mechanics. Small actions make up for big actions as they are coupled to complete goals and reward the player for their progress. It establishes a connection that is unique to the players as they feel entitled to the actions they made, while retaining their interest to further play the game, defining the ownership concept. Facilitators consist of aesthetics aspects, overall invested time, and previous experiences players had. These constructs were conceived to objectively investigate players' previous experiences and the common knowledge retained from these experiences. Game designers can investigate these constructs to tap into common knowledge to ease adaptation into their games, especially during onboarding.

Many and varying sources can form a goal: the game itself can instantiate an objective as a prerequisite to complete an activity, namely missions, quests, bounties, etc. It can also originate from players themselves depicting their personal projection onto the game and it can ultimately be impacted by social and contextual factors surrounding the experience (Calvillo-Gámez, Cairns, \& Cox, 2015; Desurvire \& Wiberg, 2010).

In-game objectives are also products from design processes, involving not only the game's narrative but the development of all steps to be completed. For example, a strike, a player versus environment (PvE) activity (player against the computer), in Destiny 2 (Bungie, 2017) seen at Figure 2, consisting of a set of objectives to be accomplished, from small actions such as pressing a button or raising a lever, to large actions such as defeating the boss commonly placed at the activity's end. The strike can also be visualized as a large flowchart, like the user's journey UX 
designers are familiar with, comprehending all the activity's complexity conceived by game designers.

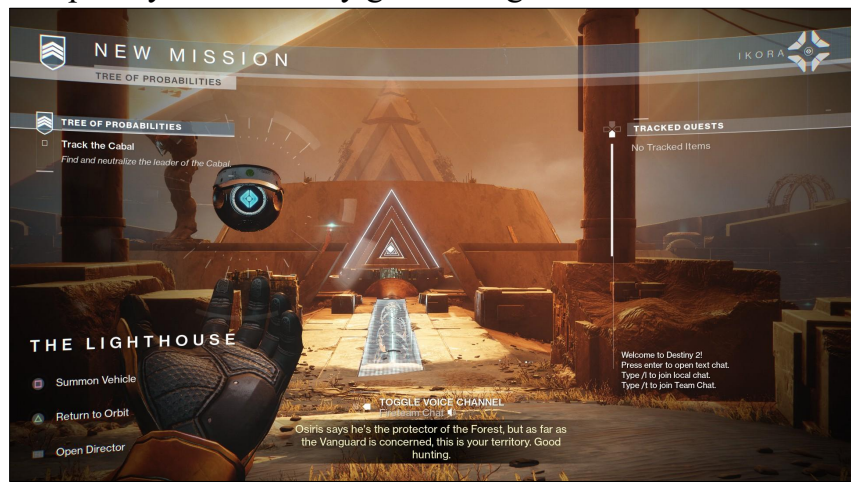

Figure 2. Current objectives shown through the game's Heads-Up Display.

As means of response and congratulation, games employ rewarding systems to compensate players for completing their missions or reaching a milestone as Desurvire and Wiberg (2010) defines reinforcement. The most common reward given in games is experience points, an abstract number representative of how experienced a player is, mainly used to determine skill levels. Other types of rewards are in-game items used to improve the player's gameplay: more powerful weapons and armor, allowing them to face more challenging activities; in-game currency, allowing them to independently purchase items sold by in-game NPCs; materials used for in-game items upgrade, allowing them to upgrade their weapons and armor at will. Figure 3 exemplifies how rewards are given through a screenshot from Overwatch (Blizzard Inc., 2016), where medals are awarded to players who achieved the highest scores throughout the gameplay, alongside experience points shown at the bottom.

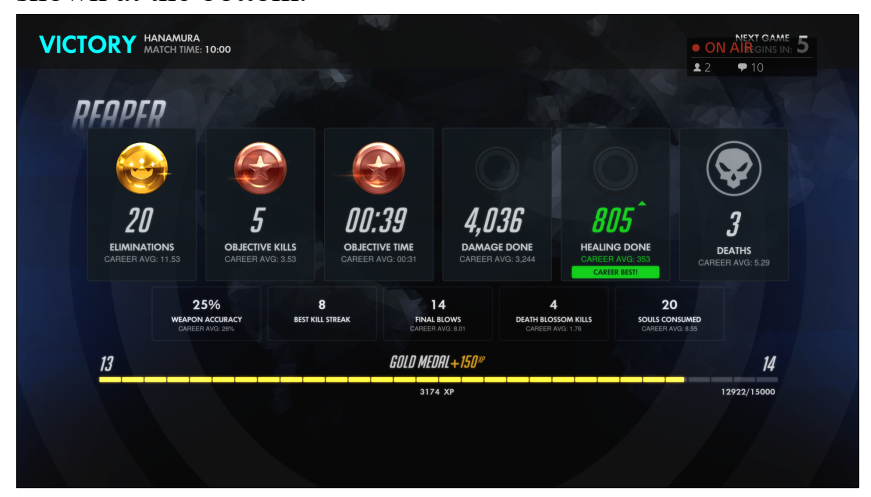

Figure 3. Session completion and rewards.

\subsubsection{Learning with Games}

Games can also be used for educational purposes aligning fun and learning to overlap during the experience exertion, establishing systems to gradually empower players as they evolve through gameplay. Learning principles refer to the mental models' players adhere to when going through experiences and how they process their learnings while facing challenges (Desurvire \& Wiberg, 2010; Champion, 2011). The learning process can be aided by several means, as Desurvire and Wiberg (2010) define within Game Approachability Principles (GAP). They are as follows: the amount of type of practice, amount of type of demonstration, reinforcement, self-efficacy, scaffolding, being in control, good design guidelines (Heuristic Evaluation for Playability - HEP), clear game goals, information on demand, and selfmastery.

Mechanics serve as rules, or rather, the domain of possible actions the game allows players to progress in both knowledge and content (Takatalo, Häkkinen, \& Nyman, 2015). A balance between skill and challenge provides a state of flow favorable to the construction of knowledge (Csikszentmihalyi, 1990), serving as a golden rule for designing mechanics: they need to be attainable and learnable. Otherwise, an imbalance between challenge and skill hinders the learning process, either because the player does not have the knowledge to support the intention they want to complete, or because the challenge has failed to offer a relevant experience (Desurvire, Caplan, \& Toph, 2003; Hodent, 2017). These two outcomes build frustration and end up repelling the player.

A good introduction to the game's mechanics can help in constructing knowledge, acting on one side of the balance of challenge versus skill. The first hour of experience with a game characterizes the onboarding process, a very important introductory step in which the game teaches the player its main mechanics, gives them space to practice it, allowing mistakes to happen and be remedied in order to build a knowledge base for future mastery. Games commonly implement this approach through tutorial sessions usually applied during the first experiences and maintain them throughout the experience using the Heads-Up Display (HUD), for example, as an aid to fixing knowledge and preventing future errors, which can be seen in Figure 4. As part of the UI, the HUD serves as a visual communication between the game and players, employing a dynamic interface adaptative to what players are seeing in-game (Hodent, 2017). In Figure 4, Fortnite's HUD (Epic Games, 2017) is shown, with a map containing the player's current location and points of interest in the virtual world at the upper right corner, the list of teammates in the upper left corner, the available slots to carry items found around the world at the lower right corner, and information about the player's character life at the lower left corner. At center, we see the HUD's full potential, a small window showing the item's information the player is aiming at, as well as the indication of a button to be pressed to pick up the item.

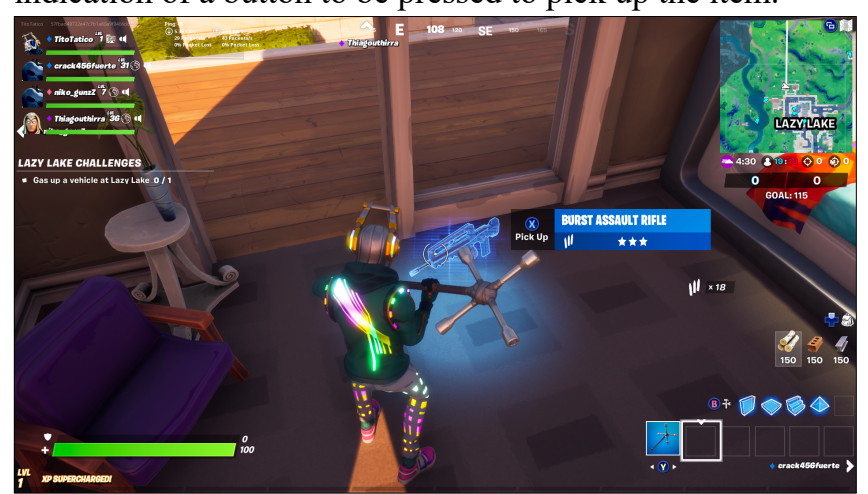

Figure 4. HUD showed in Fortnite (Epic Games).

In another work, Desurvire and colleagues (2004) define HEP by joining guidelines of game usability, game 
mechanics, game narrative, and gameplay to guide development, promoting solutions as reducing repetitive punishment due to repetitive failure, available activities variety, and adaptable pacing to minimize user's fatigue or boredom. Clear game goals evaluate the game's ability to enable the players' success in achieving their goals, and to attract and retain their interest. Information on demand evaluates how useful learned action and skills are perceived to the user throughout the game. At last, self-mastery outlines the game's ability to lead players onto mastery of the rules and mechanics of the game.

As the user progresses over time, more and more mechanics are revealed, as the advancement will prepare players and make them feel efficient as their in-game level and sense of self-efficacy will increase accordingly. The game must provide opportunities for enough practice of skills and mastery, and reinforce this aspect within each action, provided alongside feedback: a congratulating message or an achievement appropriate to the invested effort (Isbister, 2010). The learning process though may not necessarily evolve through positive outcomes but also through negative ones instead. Failure must be assigned and addressed so long as the game balances punishments to accurately convey the message that doing such action is not appropriate to the given context, which refers to feedback (Isbister, 2010).

\subsubsection{Importance of Context}

At all times, the context plays a vital role in defining how the experience is impacted (Takatalo, Häkkinen, \& Nyman, 2015). Real and virtual environments each play their respective roles in influencing context as they both hold ambiance, presence, and interfaces that tailor the experience. That said, contextual factors can originate from a series of sources. Elements such as the location players are in and its momentary aspects such as temperature, lights, sound, and smell (Cohen \& Newton Fernando, 2009), the interfaces and devices they are using to output their outcomes on the experience, and the people they are interacting with either through real or virtual conditions (Champion, 2011), are essential to evaluate the overall impact the context has in the experience.

Cohen and Fernando (2009) suggest that both the physical ambiance and the game's virtual ambiance can coexist and overlap its elements. Thus, context pertained to the virtual ambiance is also accountable and can impact the experience. Additionally, multiplayer games connect many players from different locations around the globe through the Internet, connecting to the multiple spaces and multipresence concepts defined by Cohen and Fernando (2009).

\subsubsection{Player Behavior in Cognitive Psychology}

It is known that every player is situated to have their own experiences, having clear distinctions that when put side-byside, it can trace back to even influence exerted by cultural factors (Cohen \& Newton Fernando, 2009). Differences can also be seen when focusing on gender disparities. Such disparities are observed in terms of players' perception of
NPCs' visual and attitudinal aspects (Denson et al., 2015), learning experiences established by programs designed focused on female aspects (AlSulaiman \& Horn, 2015), creation of links and personal identification between player and character (Vanderhoef, 2015), and adoption of players to games from their perceptions about the game character's gender (Bennet, 2018).

As game designers, it is necessary to understand how players perceive and interact with games, an important step to be taken towards bettering gaming experiences. The main response to how games are perceived is to investigate player behavior during their experiences. Cognitive psychology focuses on players' cognitive abilities and capabilities when joining perception, attention, memory, emotion, and motivation (Nacke, 2015; Takatalo, Häkkinen, \& Nyman, 2015; Hodent, 2017).

Perception is the first step for enticement as it is a mind construct allowing players to realize and understand the aspects of games and benefit the feelings of presence, awareness, and immersion through sensory and cognitive capabilities, namely spatial orientation, brightness, and loudness (Takatalo, Heikkinen, \& Nyman, 2015).

To retain the knowledge perceived during the player's experiences, memory plays a significant role (Hodent, 2017). The knowledge retained over time will improve the player's sense of self-efficacy and self-mastery, designed to characterize the player's sense of owning the game's mechanics (Calvillo-Gámez, Cairns, \& Cox, 2015; Desurvire \& Wiberg, 2010).

Hodent (2017) says attentional resources are scarce, turning it into a solid aspect to designing gaming experiences, especially the UI, the main tool used to communicate with players. To retain knowledge, the user must attentionally perceive the game's interaction modalities to best encode information. Developers must craft games to enable each informational aspect that is of importance to the experience on the user's reach. Too much information can distress the player rather than allow for a steady experience (Thomas, Moffat, Bailey, \& Scholer, 2014).

Csikszentmihalyi (2008) wrote that motivation could help retain players by giving appropriate rewards as reinforcement being intrinsically motivating to the player. Many factors can influence how a player feels motivated, such as intrinsic and extrinsic factors, along with explicit and implicit factors (Hodent, 2017). Developers can tune their games to influence players using extrinsic factors to motivate them in moving forward with any given objective. It can also be tied to the concept of ownership when rewarding players, as well to reinforce which actions players should take, as they are aware doing such actions can result in satisfactory outcomes (Desurvire \& Wiberg, 2010; Hodent, 2017; Csikszentmihalyi, 1990).

Emotions are often a hard topic to be tackled and are thus heavily supported by GUR methodologies (Nacke, 2013). Emotions are multifaceted, as well as the factors that may influence it, ranging from personality traits to momentaneous thoughts (Maia \& Furtado, 2019). 


\section{The GLIMPSE Framework}

\subsection{Method}

After conducting the presented literature research, an iterative process has been established to formulate the GLIMPSE framework creating elements and sub-elements. We seek to make sense of emulating the reasoning used in game design inspired by our perception of the importance of each construct in order to design a game with the potential to achieve a sense of immersion, also in conjunction with the knowledge of the authors of this article on experience with games acquired over a period of 10 years or more.

We created the framework with the following objectives in mind: i) to evaluate the games' abilities to create pleasurable experiences; ii) to gather information on the player's perception of the elements with their gaming experiences, and iii) to evaluate the immersion perceived by the user according to the framework's elements.

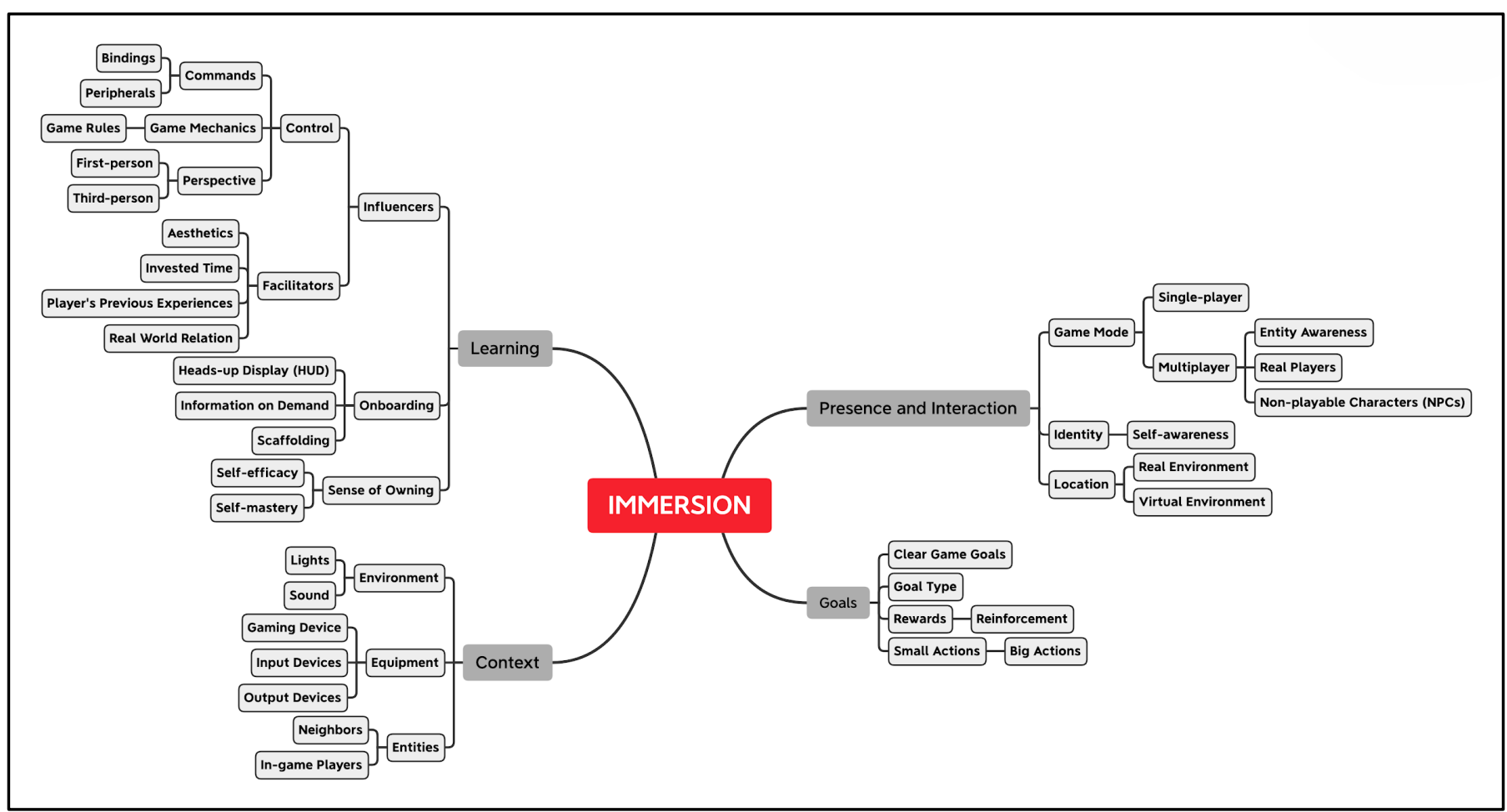

Figure 5. The GLIMPSE framework.

GLIMPSE was structured as a mental map to visually represent its main elements and sub-elements, as can be seen in Figure 5. A hierarchy is established so elements can be further described by its sub-elements, which in turn also unfold to their own sub-elements, and so forth. This design choice aims to increase the main elements' granularity to better identify their presence in gaming experiences.

\subsection{Its Elements and Representation}

The Presence and interaction main element seek to evaluate the game's ability in enabling players to feel self-aware, as well as other interactable entities enabling social interactions. These entities can be represented by real players or NPCs in single-player or multiplayer game modes. The concepts of presence and co-presence, coined by Cohen and Fernando (2009), are our main inspiration for this element, thus representing the player's perceived sense of presence in the real and virtual environments as well the sense of copresence shared with local or virtual peers.

The next main element, called Goals, evaluates the game's ability in establishing goals. In particular, the goals element evaluates how perceivable the proposed goals are to players and how likely players are to succeed in pursuing goals. At goal completion, this element evaluates if the reward is appropriate for the players' effort as reinforcement through gratification. A goal is understood to be a big action, which is defined by smaller actions when put in perspective.

The Context main element evaluates the players' perception of the impact provided by the environment during their experience, for example, television as an output device connected to a PC or video gaming console with either a gamepad or mouse and keyboard as input peripherals. It also evaluates the impact the environment characteristics have on the overall experience alongside the impact players sense when joined by other relatives.

The Learning main element evaluates the game's ability to establish learning processes involving players and their journey to self-mastery with game mechanics. It evaluates the onboarding process when the game introduces players to its mechanics. The sense of self-efficacy can be assessed using the scaffolding, information on demand, as proposed by Desurvire and Wiberg (2010), and the HUD sub-element. After the onboarding process, aspects such as overall invested time players had, as well as previous experiences with other games. Other aspects pertaining to the game such as its aesthetics, controls, and viewing perspectives are also evaluated with this element. Lastly, it evaluates the player's sense of owning the game through self-efficacy and self- 
mastery sub-elements. This element was based on the Puppetry concept from Calvillo-Gámez et al. (2015) and the GAP list from Desurvire and Wiberg (2010).

\section{Validation}

\subsection{Objectives}

In this section, we explain the validation objectives (VO) based on the literature and to better understand the impact of elements on gaming experiences. 5 validation objectives have been defined. Next, each objective will be justified on how it motivated the need to investigate the importance of each framework's main element involved in game design, further discussed in section 6 .

When thinking of triple A games like Destiny 2, Overwatch and Fortnite, we clearly see the developers' effort to reflect the real world within the virtual world. These games have as main pillar activities both $\mathrm{PvE}$ and player versus player $(\mathrm{PvP})$, established by online matches where players connect through the internet to cooperate or compete. We understand that employing the use of virtual entities, whether controlled by the game or by other players, can impact the players' sense of immersion. With that being said, we propose the following:

VO1 To investigate the participant's interaction with other agents, be them real or virtual, controlled either by AI or other players.

The games mentioned above employ complex systems that guarantee players the ability to execute actions as they see fit, offering both space and time for the mechanics practicing, leading players to reach the end of the activity. With that being said, we propose the following:

VO2 To investigate what sub-elements/aspects affect players' interaction with games' goals.

These games also employ narratives and experiences that build and enhance both player skill and in-game character evolution. A quite common example found in triple A games are skill trees, built-in systems that allow players to choose which character aspects they want to evolve, unlocking skill points as they advance in level through gaining experience points. From our experience with games, we believe player's evolution can be portrayed not only through skill trees or ingame level but also through achievements, visual changes to character's appearance thanks to powerful rewards, increased challenge gameplays, better items arsenal, and reduced time spent in doing previously completed activities. Inspired by this rationale, we propose the following:

VO3 To investigate the participant's perception of games' capacities to stimulate learning processes.

The context in which the player is inserted can vary in many aspects, from the physical presence of other players, the equipment the player is using to perform their actions ingame, what is being displayed by the game, and sensory aspects such as light and sound coming from the physical environment the player is located as well as the virtual environment. In the face of the literature and our previous knowledge in gaming experiences, we propose the following:

VO4 To investigate if contextual aspects of game design affect the player's sense of immersion.

We understand users may experience games in many ways. As per our literature review, the reasoning behind these differences can range from broad aspects such as cultural and societal to more specific ones such as age and gender. We believe gender can imply different perceptions of immersion and to the elements and sub-elements we proposed through GLIMPSE. Thus, we define the following objective:

VO5 To investigate if gaming experiences are perceived differently according to player gender.

\subsection{Questionnaire}

A questionnaire was designed to test GLIMPSE's potential in being used for evaluation purposes. The questionnaire aims to qualify each element with a diverse set of sentences, joining two or more sub-elements.

The questionnaire was conceptually envisioned as an approach to gather player experience, more specifically as if it were to be used as a post-playtest questionnaire. Divided into sections, each dedicated to each main element, the questionnaire consisted of sentences written after GLIMPSE sub-elements, in a subjective manner to prompt participants into recalling their own gaming experiences as we did not have a specific game to evaluate, nor was this the questionnaire's objective.

We believe that the questionnaire would give us an extensive view on how players understand and perceive the relevance of the sentences on their experiences with games. Especially when we write the sentences focusing on the mentioned objectives, and interweaving sub-elements, after all, we seek to achieve immersion, even if some subelements can be deemed absent.

Questionnaire's sections 2 to 6 were built according to GLIMPSE elements, aside from the first and last section (Demographic Data and Mentioned Games, respectively), displaying sentences participants must deem how strongly they agree or disagree through a Likert scale, ranging from 1 "completely disagree" to 5 "completely agree". Notably, in the immersion section, we ask how important the participants believe each aspect (e.g.: first or third person cameras, engaging narrative, game mechanics) is to their perceived sense of immersion in the game experience, maintaining the same Likert scale while changing the available responses, now ranging from 1 "not important" to 5 "very important". Displaying sentences participants must deem how strongly they agree or disagree through a Likert scale, ranging from 1 "completely disagree" to 5 "completely agree".

Lastly, we request participants to mention games they felt inspired during their time responding to the questionnaire. The questionnaire sentences can be viewed in full at Appendix I. 


\subsection{Participants}

The main participant profile we focused was with gamers in general: players who play any game despite its genre and number of hours invested in playing it. After designing the questionnaire, we proceeded to post it on gaming forums found in various internet mediums such as Reddit and Facebook. We have carefully chosen which forums to post to, aiming to gather a great number of responses while looking for diversity in nationality, game genre, and consequently the general gaming public.

\subsubsection{Responses}

The questionnaire collected 241 valid responses of which $115(47.72 \%)$ were women, and $126(52.28 \%)$ were men, with most participants (152) between 16 to 25 years old $(63.07 \%)$. Other age groups: below 16 years old $(6.22 \%), 26$ to 40 years old $(28.25 \%)$, above 41 years old $(2.48 \%)$, as shown on Figure 6.

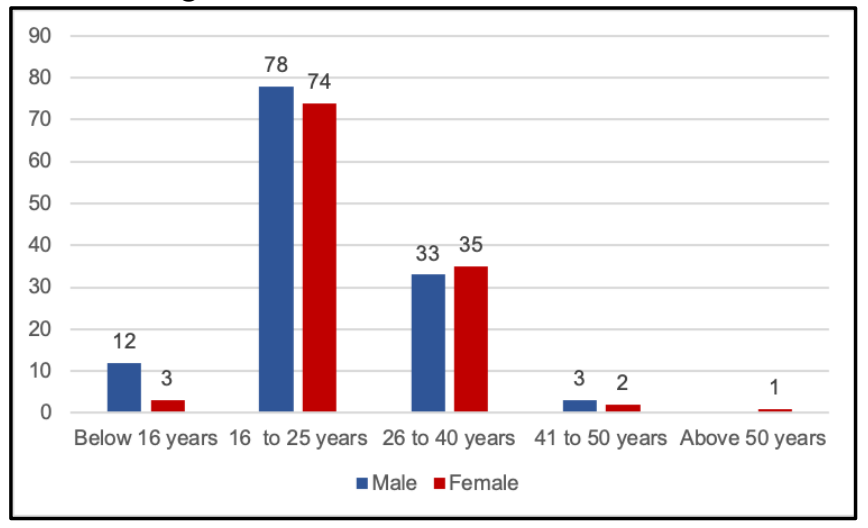

Figure 6. Participants by gender and age.

The results regarding the respondents' perception of each framework element are described in section 5. The analysis of results was made to examine the relationships between the main elements (see section 6), through Pearson's coefficient. The analysis identified correlations with statistical significance reflecting gender-wise and age-wise differences between participants.

The questionnaire was distributed in two languages: Portuguese and English with most participants being Brazilian $(n=182,75.51 \%)$. When viewing participants by Brazil's regions, a great number of respondents are from the southeastern region, followed by the northeast, south, midwest and north regions. The English version made us reach North America, the United Kingdom, Europe, and Oceania $(n=59,24.49 \%)$. The low distribution of samples from foreigners had us focusing on stratifying the data by gender and age, thus considering samples with distribution that could support plausible results.

\section{Results}

According to participants' responses, the physical and virtual environments characteristics, and sounds (92.12\%), physically or virtually present peers $(69.71 \%)$, have a considerable impact on their gaming experience. Participants have also deemed the connection between themselves and the game to be important and impactful towards their experience $(87.5 \%)$. This connection is established through the presence of entities, which can either be NPCs or virtually located players (54.8\%), game mechanics $(87.5 \%)$, realistic aesthetics $(79.7 \%)$, in-game character's control (87.41\%) though answers were tied when asked if games gave players full control and allow players to play as they please.

Participants believe goals should be completed at their will $(80.1 \%)$ while portraying clear and well-defined objectives $(80.1 \%)$ and reinforce their learning curve towards the game's mechanics mastery $(81.3 \%)$. When questioned about their liking of having more players involved in goal completion, $49.38 \%$ of participants have agreed while $31.95 \%$ disagreed, the majority were women. Also, players believe games appropriately reward them for their invested efforts $(65.15 \%)$.

Regarding the games' ability to establish learning processes, participants like games to influence their mastery journey (93.4\%), and $87.1 \%$ agree that their past experiences also influence their learning experiences. The participant's learning process shows a link to the in-game character's progression, as $77.6 \%$ agree with this. In their experiences, $25.7 \%$ of participants believe games disallow them to evolve the way they see fit.

Participants agreed that the environment they're physically located $(67.6 \%)$ and the equipment being used to play games $(80.5 \%)$ impact their experience. Physically present peers impact context as $77.5 \%$ of participants have agreed, creating emotional links between players, as $58.9 \%$ of participants have agreed.

Game mechanics (94.2\%), video, audio, game and accessibility settings (94.2\%), engaging narratives (92.9\%), clear and flexible objectives (88\%), audible and tactile responses $(83.8 \%)$, liberty to evolve $(80.9 \%)$, linked with the participants' perception of immersion, have been deemed to be the essential elements in fostering immersion. $71.4 \%$ of participants believe NPCs impact their perceived sense of immersion. Participants do not think that either first or third person cameras would impact their experience.

In the last section, participants reported the games they had in mind while answering the questionnaire. A total of 214 games were mentioned, of 21 different game genres, with top five being: Overwatch, The Sims, Destiny, Grand Theft Auto, and Fortnite. A word cloud (Figure 7) has been created to illustrate the incidence of which each game title has been mentioned. Considering these games and their respective genres, Figure 8 presents the relation between gender and game genre. 


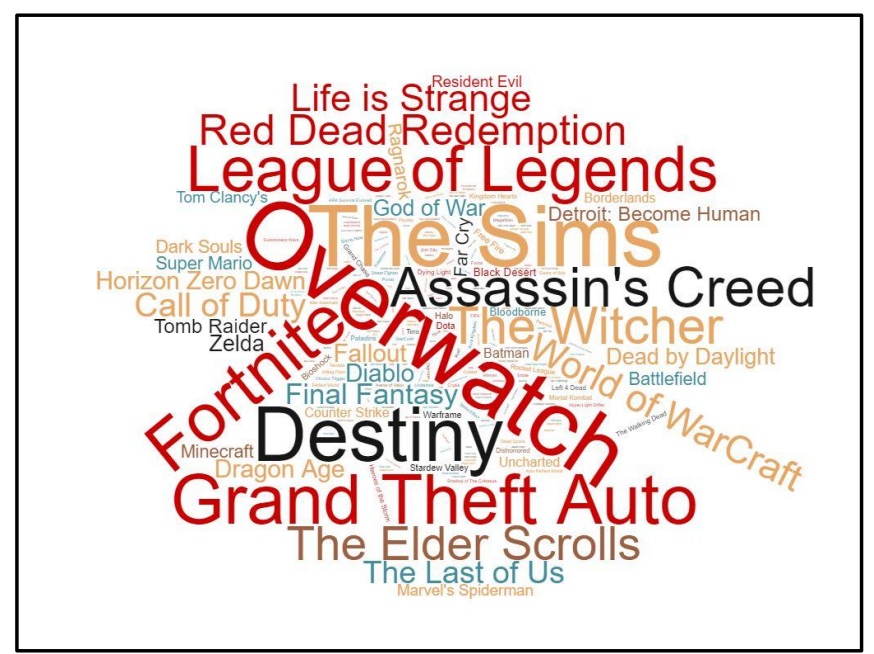

Figure 7. Word cloud depicting the listed games. The bigger it is, the more it was mentioned.

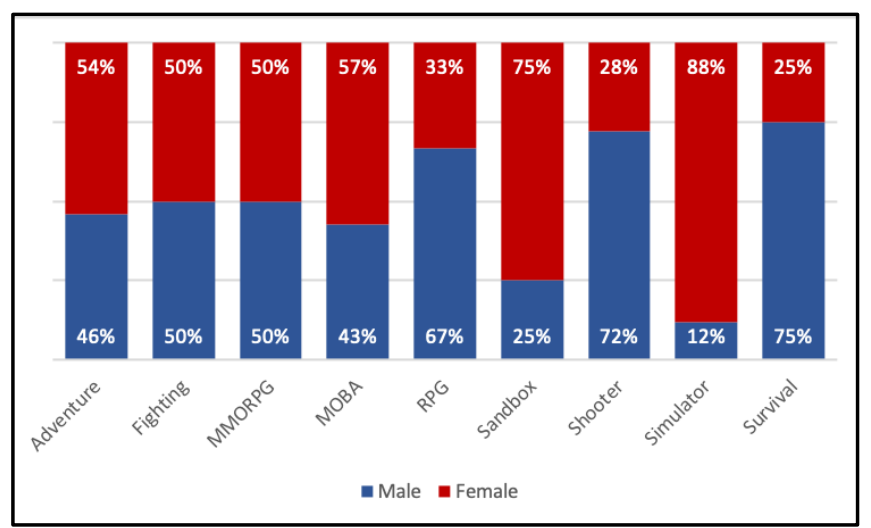

Figure 8. Game genres by gender.

\section{Analysis of Results}

We used Pearson's correlation to find relevant connections between sentences. For that, we decided on positive and negative intervals, ranging from 0.4 to 1 and -1 to -0.4 , respectively. Positive correlations mean participants' responses for two given sentences grow proportionally, while negative correlations mean participants' responses grow inversely.

We started by calculating Pearson's correlations for all 241 responses to know which sentences to further analyze. The correlation values which met the proposed intervals from any of the specified groups were also extracted from all other groups, for comparison purposes, and are shown on Table 3.

To broaden our analysis, we then take into consideration the averages and standard deviations of the correlated sentences. This approach helps understand how each group either agreed or disagreed or either deemed importance or no importance at all to the sentences. Higher averages mean great agreement among the specified group, while lower standard deviation shows a solid response.

\subsection{The Importance of other Entities for Gameplay and Immersion}

The sentences \#2.1 and \#6.10 present a general positive correlation of $.514(\mathrm{p}<.01)$, averaging $4.025( \pm 1.012)$ and $3.581( \pm 0.962)$, respectively to each sentence. Further exploring the groups, we observed both males and females have their maximum correlation values $(.78, \mathrm{p}<.01 ; .663$, $\mathrm{p}<.01$ ), respectively, in the age group from 26 to 40 yo. After analyzing the mean and standard deviation results, females (4.054, \pm 0.932$)$ have agreed better than males (3.581, \pm 0.962 ) did with NPCs being important for their experiences, especially females of age group 26 to 40 years old $(4.257, \pm 0.886)$.

Sentences \#2.3 and \#4.6 demonstrate general positive correlations of $.624(\mathrm{p}<.01), .65(\mathrm{p}<.01)$ for male, and .577 $(\mathrm{p}<.01)$ for female, with both genders maximum correlation value in the age group from 16 to 25 yo, being $.712(\mathrm{p}<.01)$ for males, and .617 for females $(\mathrm{p}<.01)$. Responses for sentences \#2.3 and \#4.6 averaged 2.969 ( \pm 1.229$)$ and 3.222 $( \pm 1.213)$ for males, while females averaged $3.313( \pm 1.3)$ and $3.67( \pm 1.168)$, respectively.

Sentences \#3.5 and \#6.11 present positive correlations, as for general respondents scoring $.474(\mathrm{p}<.01)$, and .395 $(\mathrm{p}<.01)$ for males, and $.535(\mathrm{p}<.01)$ for females. The maximum values can be seen, for both males and females, in the age group from 26 to 40 yo, being respectively .49 $(\mathrm{p}<.01)$ and $.639(\mathrm{p}<.01)$. Sentences \#3.5 and \#6.11 male respondents averaged $3.310( \pm 1.229)$ and $3.389( \pm 1.035)$ while females averaged $3.043( \pm 1.453)$ and $3.357( \pm 1.265)$, respectively.

Sentences \#5.2 and \#5.4 resulted in a positive correlation of $.430(\mathrm{p}<.01)$ for all respondents, $.362(\mathrm{p}=.11)$ for males, and $.493(p<.01)$ for females, with maximum values in male's age group from 16 to 25 years old $(.363, \mathrm{p}<.05)$ and in female's age group from 26 to 40 years old $(.595, \mathrm{p}<.01)$. Males averaged on sentences \#5.2 and \#5.4, respectively, $3.746( \pm 1.213)$ and $4( \pm 1.020)$, while females averaged $3.809( \pm 1.311)$ and $4.061( \pm 1.118)$.

Sentences \#2.4 and \#6.11 show positive correlations with female respondents only $(.410, \mathrm{p}<.01)$, while males posit neutral values, close to 0 , as shown in Table 3 . The maximum value of $.408(\mathrm{p}<.01)$ is observed among females aged between 16 and 25 yo. Although male results seem neutral, the averages for sentences \#2.4 and \#6.11, respectively, were of $3.929( \pm 1.104)$ and $3.389( \pm 1.035)$, while females scored $3.748( \pm 1.241)$ and $3.357( \pm 1.265)$.

A negative correlation has been spotted among all groups between sentences \#3.5 and \#4.6, as well as between sentences \#2.3 and \#3.5. For general responses, the correlation between sentences \#3.5 and \#4.6 scored -.436 $(\mathrm{p}<.01)$, with males scoring $-.503(\mathrm{p}<.01)$ and females -.358 $(\mathrm{p}<.01)$. The minimum values for this negative correlation are observed in males from 16 to 25 years old $(-.613, \mathrm{p}<.01)$ and females from 26 to 40 years old $(-.540, \mathrm{p}<.01)$. Males averaged $3.310( \pm 1.229)$ and $3.222( \pm 1.213)$, and females averaged $3.043( \pm 1.453)$ and $3.670( \pm 1.168)$, respectively to sentences \#3.5 and \#4.6. 
As with the correlation between sentences \#3.5 and \#4.6, general results posit a negative correlation of $-.359(\mathrm{p}<.01)$, males scored $-3.73(\mathrm{p}<.01)$, and females $-.332(\mathrm{p}<.01)$, with minimum value observed on males aged between 16 to 25 years old $(-.411, \mathrm{p}<.01)$ and females aged between 26 to 40 years old $(-.362, \mathrm{p}<.01)$. Males averaged a score of 2.960 $( \pm 1.229)$ and $3.310( \pm 1.229)$ while females scored 3.313 $( \pm 1.300)$ and $3.043( \pm 1.453)$, respectively to sentences \#3.5 and \#4.6.

\subsection{Learning with Games}

The sentences \#4.1 and \#4.2 scored a positive general correlation of $.405(\mathrm{p}<.01)$, while males showed a correlation of $.203(\mathrm{p}<.05)$, and females $.590(\mathrm{p}<.01)$, with maximum value observed on males between 26 to 40 years old (.439, p.05), and females between 16 to 25 years old $(.590, \mathrm{p}<.01)$. For sentences \#4.1 and \#4.2, respectively, males averaged $3.587( \pm 0.998)$ and $4.437( \pm 0.699)$, while females averaged $3.713( \pm 1.122)$ and $4.617( \pm 0.720)$.

\subsection{Environment Impact on Immersion}

The sentences \#2.7 and \#6.4 present a positive general correlation of $.443(\mathrm{p}<.01), .334(\mathrm{p}<.01)$ for males, and .512 $(\mathrm{p}<.01)$ for females, with maximum value observed on males between 26 to 40 years old $(.334, \mathrm{p}>.05)$, and females between 16 to 25 years old $(.525, \mathrm{p}<.01)$. Males averaged $3.897( \pm 1.172)$ and $3.897( \pm 1.104)$ for sentences \#2.7 and \#6.4, while females averaged $4.365( \pm 0.949)$ and 4.365 $( \pm 1.241)$, respectively.

Sentences \#2.9 and \#5.2 scored a positive general correlation of $.654(\mathrm{p}<.01), .664$ for males, and .645 for females. Maximum values were almost tied for both male age groups between 16 and 25 and 26 to 40 yo, with a difference of -.028 . Females have a higher coefficient in the 16 to 25 years old age group $(.684, \mathrm{p}<.01)$. For sentences \#2.9 and \#5.2, respectively, males scored a mean of 3.651 $( \pm 1.310)$ and $3.746( \pm 1.213)$, while females averaged 3.748 $( \pm 1.343)$ and $3.809( \pm 1.311)$.

\subsection{Interactions and Adjustable Settings}

The sentences \#6.12 and \#6.13 present a positive general correlation of $.291(\mathrm{p}<.01)$, with $.141(\mathrm{p}<.01)$ for males, and $.460(\mathrm{p}<.01)$ for females. Males between 16 to 25 years old scored the highest coefficient between the male age groups $(.199, \mathrm{p}<.01)$. It is important to mention that males between 26 to 40 years old scored a low of $.029(\mathrm{p}<.05)$, pointing a difference of .17. Meanwhile, females aged between 16 to 25 years old scored a coefficient of $.540(p<.01)$, showing a great difference between genders, even if compared to females aged between 26 to 40 years old $(.343, \mathrm{p}<.01)$.

Table 3. Correlations on groups by gender and age

\begin{tabular}{|c|c|c|c|c|c|c|c|c|c|}
\hline \# & Sentence 1 & Sentence 2 & All & M & $16-25$ & $26-40$ & F & $16-25$ & $26-40$ \\
\hline 1 & $\begin{array}{l}2.1 \text { NPCs are important fo } \\
\text { gameplay }\end{array}$ & $\begin{array}{l}\text { r } 6.10 \text { Presence of other NPCs } \\
\text { impacting immersion }\end{array}$ & $.514 * *$ & $.486^{* *}$ & .277 & $.785^{* *}$ & $.540 * *$ & $.476^{* *}$ & $.663^{* *}$ \\
\hline 2 & $\begin{array}{l}2.3 \text { Playing alone } \\
\text { allows for more } \\
\text { interactions }\end{array}$ & $\begin{array}{l}\text { 4.6 Evolving Better While } \\
\text { Playing Alone }\end{array}$ & $.624 * *$ & $.650 * *$ & $.712 * *$ & $.486^{* *}$ & $.577 * *$ & $.617^{* *}$ & $.407^{* *}$ \\
\hline 3 & $\begin{array}{l}2.7 \text { Realistic virtual } \\
\text { environment reinforces } \\
\text { presence }\end{array}$ & $\begin{array}{l}\text { 6.4 Realistic Visual } \\
\text { Aesthetics impacting } \\
\text { immersion }\end{array}$ & $.443 * *$ & $.334 * *$ & $.285^{* *}$ & .334 & $.512 * *$ & $.525^{* *}$ & $.382^{* *}$ \\
\hline 4 & $\begin{array}{l}\text { 2.9 Physical environment } \\
\text { impacts game interaction }\end{array}$ & $\begin{array}{l}\text { 5.2 Physical environment } \\
\text { impacts immersion }\end{array}$ & $.654 * *$ & $.664 * *$ & $.658^{* *}$ & $.686^{* *}$ & $.645^{* *}$ & $.684 * *$ & $.538^{* *}$ \\
\hline 5 & $\begin{array}{l}3.5 \text { Games involving more } \\
\text { players to complete goals }\end{array}$ & $\begin{array}{l}6.11 \text { Virtual presence of other } \\
\text { players }\end{array}$ & $.474 * *$ & $.395 * *$ & $.444 * *$ & $.490 * *$ & $.535^{* *}$ & $.478^{* *}$ & $.639^{* *}$ \\
\hline 6 & $\begin{array}{l}\text { 5.2 Physical environment } \\
\text { impacts immersion }\end{array}$ & $\begin{array}{l}\text { 5.4 Physical presence of } \\
\text { others impact context }\end{array}$ & $.430 * *$ & .362 & $.363^{*}$ & $.358 * *$ & $.493 * *$ & $.442 * *$ & $.595^{* *}$ \\
\hline 7 & $\begin{array}{l}\text { 6.12 Sensorial feedback } \\
\text { impacting immersion }\end{array}$ & $\begin{array}{l}6.13 \text { In-game settings } \\
\text { impacting immersion }\end{array}$ & $.291 * *$ & $.141^{* *}$ & $.199 * *$ & $.029^{*}$ & $.460 * *$ & $.540 * *$ & $.343 * *$ \\
\hline 8 & $\begin{array}{l}\text { 4.1 Likeness to play } \\
\text { games to learn new } \\
\text { content }\end{array}$ & $\begin{array}{l}4.2 \text { Likeness of games } \\
\text { which induce player } \\
\text { evolution }\end{array}$ & $.405^{* *}$ & $.203^{*}$ & $.148^{*}$ & $.439^{*}$ & $.590 * *$ & $.614^{* *}$ & $.372^{* *}$ \\
\hline 9 & $\begin{array}{l}2.4 \text { Able to interact even } \\
\text { in a virtual condition }\end{array}$ & $\begin{array}{l}6.11 \text { Virtual presence of } \\
\text { other players }\end{array}$ & $.261 * *$ & .074 & $.077^{*}$ & -.001 & $.410 * *$ & $.408^{* *}$ & $.350^{* *}$ \\
\hline 10 & $\begin{array}{l}\text { 3.5 Games involving } \\
\text { more players to complete } \\
\text { goals }\end{array}$ & $\begin{array}{l}\text { 4.6 Evolving Better While } \\
\text { Playing Alone }\end{array}$ & $-.436^{* *}$ & $\begin{array}{l}-.503 \\
* *\end{array}$ & $\begin{array}{l}-.613 \\
* *\end{array}$ & $\begin{array}{l}-.175 \\
* *\end{array}$ & $\begin{array}{l}-.358 \\
* *\end{array}$ & $\begin{array}{l}-.221 \\
* *\end{array}$ & $\begin{array}{l}-.540 \\
* *\end{array}$ \\
\hline 11 & $\begin{array}{l}2.3 \text { Playing alone allows } \\
\text { for more interactions }\end{array}$ & $\begin{array}{l}3.5 \text { Games involving more } \\
\text { players to complete goals }\end{array}$ & $-.359 * *$ & $\begin{array}{c}-.373 \\
* * \\
\end{array}$ & $\begin{array}{c}-.411 \\
* * \\
\end{array}$ & $\begin{array}{l}-.278 \\
* *\end{array}$ & $\begin{array}{c}-.332 \\
* * \\
\end{array}$ & $\begin{array}{l}-.257 \\
* * \\
\end{array}$ & $\begin{array}{c}-.362 \\
* * \\
\end{array}$ \\
\hline
\end{tabular}




\section{Discussion}

Presence and interaction in games. Correlation 2 (see Table 3) points out that participants agree that playing alone allows them more interactions and makes them feel that they evolve in the game even better. These results bring evidence that this may be related to the fact that when playing alone, players can define what activities they would like to perform, when and how they want to perform it without having to be grouped with other players. With this autonomy, players can choose how they evolve and what they do in the game. If the user intended to play in group activities, they would be aware that another entity would influence their actions, and they might need to evolve their strategy jointly with this entity. If players seek this end, they consciously decided to play a game that requires a group strategy to achieve an objective.

From correlations 5, 10, and 11, all sharing sentence \#3.5, participants demonstrate they can interact in a virtual environment, and the presence of other characters controlled by other players impacts their immersion, which completes VO1. Taking the games mentioned as an example, with group activities being an intrinsic part of their design, promoting team strategy, providing communication between players, and allowing a series of agents to influence the course of the game, whether playing as allies or opponents.

The sense of presence is not limited to virtual characters controlled by other players. NPCs can perform similar functions with intelligence that shows autonomy and personality. NPCs are often used to build a history and play roles of supporting characters. Games such as Overwatch and Destiny have stories representing fictional universes, where it is possible to interact with NPCs, get to know about their past, feel like authors of their present and think about their futures. This impacts the player's sense of immersion by bringing experiences that go through a player-centered story enabling them the protagonists and storytellers' roles. The protagonism fosters the autonomy and ownership feelings for the performed actions, as well as recognizing the possible consequences as they unfold.

Freedom to complete and rewards that build engagement. In open world games, especially the most mentioned games from the questionnaire, completing an objective seems to be a limitless task. As we proposed VO2, aspects such as clear game goals and appropriate rewards reinforce the players' interaction and engagement with games. Having the power to define the order and which objectives to complete gives the player freedom to explore the narrative and practice the mechanics of the game. An appropriate reward ensures that the player feels motivated to continue playing with better items that enhance their gaming experience.

To Learn and Evolve. From a chronological perspective, games establish a learning process, commonly represented by a curved line, where the player's mastery increases with the invested time. The onboarding process through tutorials helps players start learning and get ready for more complex experiences, taking advantage of the best the game has to offer, completing objectives and earning points to evolve their character. These aspects are perceived by the participants as important for their interaction with games and their perception of their own evolution as a player, which satisfies VO3.

Realistic virtual environments impacting immersion. As in other market products, there is an abstraction of what is real to what can be represented in a system; for example: in a database, there are entities with their defining attributes that refer to practical life aspects. As virtual environments are a crucial feature of games, it is possible to bring them closer to reality by drawing connections between what is seen in the real environment and the virtual environment. The abstraction from the real world to the virtual one comes to be so minimal that several games employ the use of graphics engines that even simulate Ray Tracing, resulting in absurd photorealism. These graphics engines bring the game closer to an almost parallel reality, being as rich and visually pleasurable as real life. Virtual landscapes, with mountains, forests, rivers, and oceans, show scenarios that, for some, are impossible to visit.

There is realism beyond the visual aspect. The virtual environment can also completely mimic aspects of a real environment: ranging from houses, streets, buildings, trees, forests, cars, fences, any objects found in the real world, or that comes to the developers' minds. With this level of customization, it is possible to add more realism by bringing functional features that make up the objects of the virtual world. A battle tank doesn't just have to look like a battle tank, it needs to behave as such: moving slowly, due to its weight, having a great defense against substantial damage and being able to crush or destroy any obstacles in front of it. These functional characteristics enrich the virtual world and, consequently, the players' gameplay as well, bringing ways to execute their actions and strategies more realistically. This, in turn, satisfies VO4 by clarifying which aspects sustain immersion through contextual aspects.

Gender disparities. As other studies have widely reported, gender disparities are also part of gaming experiences as humans interact through it after the image of our society. This is to say the same patterns of gender disparities observed in areas outside gaming experiences have come to impact it. Considering VO5, we discuss gender disparities found in games in the following lines.

To begin with, the participants' responses to the games they mentioned, and their preferred game genres shows women prefer sandbox and simulation genres. The games categorized under this genre are usually designed with minimal limitations, having slower rhythms, making challenges and actions performable at player's will. Men, on the other hand, prefer game genres such as survival, shooter, and RPG, which are strategy-oriented games, requiring quick thinking at a constantly challenging pace. 
These genres are considered intense and can provide moments of high tension and are commonly played by men more than women, also according to our results.

Additionally, correlations 8 and 9 (see table 3) not only present greater correlations among women than men, but their sentences' medians, which are greater than or equal to 4, show how women are fonder of games that make them evolve as players, and how they feel the presence of characters controlled by other players impact their immersion. As much as women choose to play group activities, they do it virtually, without needing to be physically present around other players. The analysis of the results clearly shows that this scenario is not the same for men.

This gives us motive to think how game designers can drive impact to minimize gender disparities. To begin with, as a societal aspect, the team behind development must take into consideration the existence of such disparities and must be invested into facing these disparities (Heringer et al., 2019). In recent years, games have been focusing on inclusion and giving as much attention and detail to female characters as male ones receive. Females have turned to be protagonists of critically acclaimed success titles such as Horizon Zero Dawn, Life is Strange, Control, and have also been represented in bigger and bolder roles as NPC characters within games. This progress represents how strong and bold women can be inside and outside the gaming environment and its derivatives.

Although, as shown by our analysis results, when tackling social interactions within games women have responded they prefer to play alone. This becomes clear when noticing how players, as humans in a society, are to replicate the same disturbing behavior that pulls women farther from pleasurable gaming experiences. As one study found out (Kasumovic \& Kuznekoff, 2015), male players with low gaming skill tend to react aggressively towards females, while portraying a more submissive behavior to their male counterparts. On the other hand, male players with high gaming skills tend to be friendly towards women.

Despite the fact developers have implemented solutions such as reporting systems, blocking and avoiding functionalities, strict in-game punishments as means to diminish the toxic behavior within gaming communities towards players of all genders, there's much to be done as a society to educate ourselves about gender equity and envision game design approaches to empower women and other attacked minorities to be valued and have an efficient and operable defense mechanism.

\section{Conclusion}

Our initial perspective was to develop a tool game developers could tap in while designing their games, taking into consideration as many sub-elements from the framework as possible. We did not aim for any specific game or game genre when designing the framework as we wanted to see how far it would reach in terms of game genre diversity. Our main objective, though, was to share it among players of triple A games which yet holds a great genre variety. With collecting over 214 different games and 21 different game genres, we believe GLIMPSE can serve as a tool to envision game design practices and insights for different game genres with many purposes such as social online interaction, learning and serious games.

Our analysis shows that the male and female stereotypes found in our society are replicated in the social sphere of games. This can be considered as a game designers' responsibility and some have already developed solutions to minimize the impact of these stereotypes on their gaming communities. A game developed with a focus on social inclusion, especially having a team that represents inclusive social profiles, ensures that the game development will shed light in the inclusion of minorities, once developers will be intimately connected with these profiles.

This article contributes with the following study fields: HCI, a framework to aid game designers in carrying out their development approaches, serving as an extra work tool. The elements created within it bring insights on how game designers can tweak their game's mechanics, especially with the questionnaire we designed, a tool that contributes to GUR field, which we hope may inspire game designers in creating their questionnaires for playtesting, in light of the results and correlations we presented in this paper, leading to more significant findings to be analyzed.

In the future, we hope to extend our studies and adapt the GLIMPSE framework to the evolving gaming interactive mediums such as the use of voice modality interaction.

\section{References}

AlSulaiman, S., \& Horn, M. S. (2015). Peter the Fashionista? Proceedings of the 2015 Annual Symposium on Computer-Human Interaction in Play CHI PLAY '15, 185-195. https://doi.org/10.1145/2793107.2793127

Bernhaupt R. (2010) User Experience Evaluation in Entertainment. In: Bernhaupt R. (eds) Evaluating User Experience in Games. Human-Computer Interaction Series. Springer, London. https://doi.org/10.1007/9781-84882-963-3_1

Bennett B. (2018) Gaming representation: race, gender, and sexuality in video games, Feminist Media Studies, 18:5, $959 \quad$ - 961, https://doi.org/10.1080/14680777.2018.1498144

Calvillo-Gámez E., Cairns P., Cox A. (2015) Assessing the Core Elements of the Gaming Experience. In: Bernhaupt R. (eds) Game User Experience Evaluation. HumanComputer Interaction Series. Springer, Cham. https://doi.org/10.1007/978-3-319-15985-0_3

Cohen M., Newton Fernando O.N. (2009) Awareware: Narrowcasting Attributes for Selective Attention, Privacy, and Multipresence. In: Markopoulos P., De 
Ruyter B., Mackay W. (eds) Awareness Systems. Human-Computer Interaction Series. Springer, London. https://doi.org/10.1007/978-1-84882-477-5_11

Csikszentmihalyi, M., \& Csikzentmihaly, M. (1990). Flow: The psychology of optimal experience (Vol. 1990). New York: Harper \& Row.

Denson, T. F., Dixson, B. J. W., Tibubos, A. N., Zhang, E., Harmon-Jones, E., \& Kasumovic, M. M. (2020). Violent video game play, gender, and trait aggression influence subjective fighting ability, perceptions of men's toughness, and anger facial recognition. Computers in Human Behavior, 104. https://doi.org/10.1016/j.chb.2019.106175

Desurvire, H., Caplan, M., \& Toth, J. A. (2004). Using Heuristics to Evaluate the Playability of games. In Conference on Human Factors in Computing Systems Proceedings. https://doi.org/10.1145/985921.986102

Desurvire H., Wiberg C. (2010) User Experience Design for Inexperienced Gamers: GAP - Game Approachability Principles. In: Bernhaupt R. (eds) Evaluating User Experience in Games. HumanComputer Interaction Series. Springer, London. https://doi.org/10.1007/978-1-84882-963-3_8

Emmerich, K., Ring, P., \& Masuch, M. (2018). I'm glad you are on my side: How to design compelling game companions. CHI PLAY 2018 - Proceedings of the 2018 Annual Symposium on Computer-Human Interaction in Play, https://doi.org/10.1145/3242671.3242709 153-162.

Heringer, A. M. T., Pimenta, M. S., Ferreira, N. B., Domingues, D. G., \& Paulo, S. (2019). Protagonismo transgênero em jogos comerciais. Proceedings of SBGames 2019, 253-259.

Hodent, C. (2017). The Gamer's Brain: How Neuroscience and UX Can Impact Video Game Design. Retrieved from https://books.google.com.br/books?id=JzyhDwAAQB AJ

Isbister K. (2010) Enabling Social Play: A Framework for Design and Evaluation. In: Bernhaupt R. (eds) Evaluating User Experience in Games. HumanComputer Interaction Series. Springer, London. https://doi.org/10.1007/978-1-84882-963-3_2

Kasumovic, M. M., \& Kuznekoff, J. H. (2015). Insights into sexism: Male status and performance moderates female-directed hostile and amicable behaviour. PLoS ONE, 10(7), 1-14. https://doi.org/10.1371/journal.pone.0131613

Kumar J. (2013) Gamification at Work: Designing Engaging Business Software. In: Marcus A. (eds)
Design, User Experience, and Usability. Health, Learning, Playing, Cultural, and Cross-Cultural User Experience. DUXU 2013. Lecture Notes in Computer Science, vol 8013. Springer, Berlin, Heidelberg. https://doi.org/10.1007/978-3-642-39241-2_58

Maia, C. L. B., \& Furtado, E. S. (2019). An Approach to Analyze User's Emotion in HCI Experiments Using Psychophysiological Measures. IEEE Access, 7, 3647136480. https://doi.org/10.1109/ACCESS.2019.2904977

Nacke L. (2015) Games User Research and Physiological Game Evaluation. In: Bernhaupt R. (eds) Game User Experience Evaluation. Human-Computer Interaction Series. Springer, Cham. https://doi.org/10.1007/978-3319-15985-0_4

Nacke, L. E. (2013). An Introduction to Physiological Player Metrics for Evaluating Games. Game Analytics, 585-619. https://doi.org/10.1007/978-1-4471-47695_26

Oulasvirta A. (2009) Social Inference Through Technology. In: Markopoulos P., De Ruyter B., Mackay W. (eds) Awareness Systems. Human-Computer Interaction Series. Springer, London. https://doi.org/10.1007/978-1-84882-477-5_5

Sirani, J. (2019). Where Switch, PS4 Rank Among the Best-Selling Video Game Consoles of All Time. Retrieved March 30, 2020, from IGN website: https:/www.ign.com/articles/2019/10/30/top-15-bestselling-video-game-consoles-of-all-time

Takatalo J., Häkkinen J., Nyman G. (2015) Understanding Presence, Involvement, and Flow in Digital Games. In: Bernhaupt R. (eds) Game User Experience Evaluation. Human-Computer Interaction Series. Springer, Cham. https://doi.org/10.1007/978-3-319-15985-0_5

Tamassia, M., Raffe, W., Sifa, R., Drachen, A., Zambetta, F., \& Hitchens, M. (2016). Predicting player churn in destiny: A Hidden Markov models approach to predicting player departure in a major online game. IEEE Conference on Computational Intelligence and Games, CIG, https://doi.org/10.1109/CIG.2016.7860431

Thomas, P., Moffat, A., Bailey, P., \& Scholer, F. (2014). Modeling decision points in user search behavior. Proceedings of the 5th Information Interaction in Context Symposium, IIiX 2014, 239-242. https://doi.org/10.1145/2637002.2637032

Vanderhoef, J. (2015). Gaming at the Edge: Sexuality and Gender at the Margins of Gamer Culture. Communication, Culture and Critique, 8(4), 632-634. https://doi.org/10.1111/cccr.12115 


\section{Appendix I - Questionnaire Sentences}

Section and Objective
Demographic Data
Presence and Interaction
Refers to the participant's
interaction with other
agents, be them real or
virtual, controlled by
people or artificial
intelligence in the game.

\section{Goals}

Refers to the participant's interaction with the game's goals.

\section{Learning}

Refers to the participant's perception of the game's capacity to stimulate learning, providing an evolution to the player's abilities through their learning process.

\section{Context in Real and} Virtual Environments Refers to the physical and virtual environments for when the player plays games.

\section{Immersion in Games}

Refers to how connected and involved participants perceive themselves according to select gameplay aspects.
Questions and Sentences

The participant's gender, age group, and country (English version) or state (Portuguese version).

1. I feel Non-Playable Characters (NPCs) are important for gameplay.

2. To me, it doesn't matter if I have an NPC or a real player playing in a group with me.

3. I feel that playing alone allows me more interactions, as I'm free from any connections with other players.

4. I can talk and interact with other players normally, even if we're in a virtual environment.

5. As I walk through the gaming world, I feel like I'm under full control of the character.

6. I feel connected with the game's mechanics.

7. A realistic virtual environment reinforces my feeling of being present in the game world.

8. Listening to sounds from the game environment reinforces my sense of being present in the game world.

9. The environment in which I'm physically located impacts on my interaction with the game.

10. I feel that the game gives me full control so I can do as I please.

1. I think games should have objectives that reinforce my learning of their mechanics.

2. I like games that allow me to set the order of goals I want to complete.

3. The game goals must be clear and well defined.

4. I like games that allow me to complete goals the way I see fit.

5. I like games that involve more players to complete a goal.

6. I like games that change goals according to context.

7. I think games reward me appropriately for the goals I accomplish.

1. I play games that make me learn new content.

2. I like games that make me evolve during my gameplay.

3. I believe that my past experiences influence the way I play.

4. I feel that I evolve as a player as the character evolves as a character.

5. The games allow me to evolve the way I see fit.

6. I feel I evolve better when I play alone.

7. I feel I'm in the power to evolve within the virtual world of the game.

1. When I'm playing with a group, I feel like everyone is emotionally connected.

2. The environment in which I'm physically located impacts my immersion.

3. I feel that the virtual environment overlaps with the real environment.

4. The presence of others who are physically located around me impacts the context in which I'm involved.

5. The equipment I use to play impacts on the context I'm involved with.

1. Engaging narrative.

2. First Person Camera.

3. Third Person Camera.

4. Realistic visual aesthetics.

5. Game mechanics.

6. Tutorials to help me as I need them.

7. A platform that allows me to evolve the way I see fit.

8. Open world and free roaming.

9. Clear and flexible objectives.

10. Presence of other Non-Player Characters (NPCs).

11. Presence of characters controlled by other players.

12. Audible and tactile responses to interactions.

13. Video, audio, game, and accessibility settings. 